\title{
Effective Treatment against SARS-Cov-2 with Amantadine Hydrochloride
}

\author{
Włodzimierz Bodnar ${ }^{1}$, Gonzalo Emiliano Aranda-Abreu² and Albert Cortés-Borra ${ }^{3 *}$ \\ ${ }^{1}$ Specialist in lung diseases, Paediatrician at NZOZ Optima in Przemyśl, Poland \\ ${ }^{2}$ Instituto de Investigaciones Cerebrales, Universidad Veracruzana, Xalapa, Veracruz, México \\ ${ }^{3}$ CAP Vila de Gràcia - Cibeles, Institut Català de la Salut, Barcelona, Spain
}

\begin{abstract}
Based on 100 documented cases treated with amantadine, we suggest that with this method of treatment, at least $90 \%$ of severe cases in Poland (or even worldwide) can be stabilized within 48-72 hours, followed by a recovery period of several more days.

Thanks to its wide availability and the use of this well-tested drug, it will stop the disease before it causes significant damage. It would also significantly change the way we are fighting the pandemic right now. Having an effective and safe drug changes everything.
\end{abstract}

\section{Keywords}

Amantadine, SARS-CoV-2, COVID-19, Amantadine hydrochloride

\section{Introduction}

If we assume that we are looking for an antiviral drug that works directly against SARS-Cov-2, then in this case, amantadine is completely useless. If we believe that amantadine or some other drug will significantly alter the growth conditions of the virus, further research is needed. Viruses must have a well-defined host in which they "multiply". We want to emphasize that a virus can only grow under the right conditions.

It has been investigated how amantadine works in general, and also how it can potentially work in the case of a SARSCoV-2 infection [1-3]. However, for a health care professional, the most important thing is to have a drug that changes the conditions under which a virus develops in a human cell so that it cannot spread. In this way, we can save the health and life of a patient.

After reaching the appropriate concentration of the drug, we act on the virus, inactivating it at each stage of the disease. In other words, in each case, we work on the cause of the disease, i.e. the virus, by eliminating it from the body (deactivation), either in the initial period of headaches or in the last stage of pneumonia. It does not matter if the patient is 30 or 85 years old or has comorbidities or not. The principle of the approach is the same, and that is to deactivate the virus.

\section{Deduction process}

To be more specific, our experiences and further research have focused solely on amantadine hydrochloride (amantadinihydrochloridum), which we still refer to as "amantadine" for reading purposes, which may or may not be an essential aspect of treatment. Further research is needed.

Focusing now on practice: Our observations of the 30year period of treatment of viral diseases were useful, and, unfortunately, the last 90 days of the dynamically growing number of COVID-19 cases in Poland "helped" me even more to collect data.

Initial attempts at treatment, such as for the flu, may be insufficient because the symptoms of COVID-19 [4] last much longer, i.e., 4-9 days ( $2 \times 100 \mathrm{mg}$ of amantadine). Studies have been conducted on the possible positive influence of amantadine on COVID-19 [5]. Thus, in most cases, there

*Corresponding author: Albert Cortés Borra, CAP Vila de Gràcia - Cibeles, Institut Català de la Salut, Barcelona, Spain

Accepted: December 23, 2021

Published online: December 27, 2021

Citation: Bodnar W, Aranda-Abreu GE, Cortés-Borra A (2021)

Effective Treatment against SARS-Cov-2 with Amantadine Hydrochloride. Res Rev Infect Dis 4(2):156-159 
was a dramatic improvement after two days, followed by a stabilization on the first day of treatment. Therefore, different doses of amantadine in our experience have shown excellent results.

After two days, most patients had no major symptoms of the disease, except for the occasional slightly tired cough. Therefore, we came to the premature conclusion that complete deactivation of the virus takes place within 72 hours, which is not necessarily true. We emphasize that this is only my guess, as science will speak for itself in the future.

However, knowing the dynamics of the virus, it is safer to maintain prophylactic treatment until the body is sufficiently immune if there is a relapse of the disease. In a small percentage, amantadine can cause early arrhythmogenic symptoms. If side effects occur, treatment should be discontinued completely, regardless of stage. Therefore, daily and constant contact with a physician is key during this treatment.

\section{When the disease progresses tremendously}

When the patient's medical history shows that the progress of the disease is very dynamic and acute (the symptoms begin to increase hour by hour), we can suspect that, in extreme cases, the patient may be at risk of dying within a few hours. Seeing the effects of the therapy, we decided to run against time and introduce one more change in the treatment to shorten the time to reach the saturated therapeutic level as much as possible.

In this model, we already have a lot of feedback where patients literally "stand up" in a short period of time.

\section{Why is rapid treatment and additional recom- mendations important?}

If treated quickly, we can avoid disability or irreversible changes in the body, as well as complications or long-term treatment of other diseases, for example, pneumonia.

In addition to taking amantadine, a symptomatic treatment is recommended for example fever. As in any illness, it is up to the physician to decide on the antibiotic if the virus has damaged the internal organs or during regeneration and treatment of the consequences of COVID-19. Time is essential, because the virus causes great damage to the body. Please note that the recovery process may depend on how late you start treatment with this program.

\section{Patients and Methodology}

This study included a sample of 100 patients, of different ages, who presented symptoms compatible with SARS-Cov- 2 . The information was obtained from patients who were seen at my clinic from July to November 2020.

The report followed the procedure for observational studies in epidemiology (STROBE) [6].

The inclusion criteria were as follows:

- Patient's consent to participate in the study.

- Desire to Participate.
- Female or male with SARS-Cov-2 compatible symptoms.

The exclusion criteria.

- Disability.

- Medical or psychological condition, which would not allow you to participate.

- Mechanical ventilation.

\section{Patients studied}

Outpatients of the city of Podkarpackie Voivodeship Poland.

\section{Objectives of the study}

The main objective of this study was to determine whether the described treatment with amantadine would be effective in the treatment of SARS-Cov-2 infection.

\section{Scheme of treatment with different doses of Amantadine}

Treatment 1 - Disease symptoms without severe progression: Amantadine $4 \times 100 \mathrm{mg}$ (meaning 1 time every 6 hours) for 48 to 72 hours. The dose is reduced depending on whether the patient's condition is stabilized or not. Later on, the dose is to be reduced to $2 \times 1-100 \mathrm{mg}$. There is no need to administer large doses for a long period of time.

Treatment 2 - Acute and severe course of disease: When the course of the disease is very acute, its progression is dynamic from the first hours after contraction of the disease, and the patient's condition deteriorates hour by hour. In my opinion, this means we are fighting against time, where the patient's condition can progress into being very severe.

- 0 hours from contraction - first dose.

- 2 hours from contraction - second dose.

- Subsequent doses administered every 6 hours, administered for 48 to 72 hours, depending on the patient's condition.

Subsequently, the dose is to be reduced according to the scheme.

Treatment 3 - Elderly people suffering from lowered metabolic rate: When the metabolic rate is lower than normal, the patient is to be administered $100 \mathrm{mg}$ of amantadine hydrochloride every 8 hours for 2 to 3 days. Subsequently, the dose is to be reduced according to the scheme.

Treatment4 - Patient over 75 years of age, symptom-free, with a positive test result only: Recommended amantadine treatment $2 \times 1-100 \mathrm{mg}$ - preventively for 14 days.

\section{Treatment5 - Hospitalisation:}

1. Zero hour - $100 \mathrm{mg}$.

2. After an hour - $100 \mathrm{mg}$.

3. Then every 6 hours, as the therapeutic doses are high enough - administered for 48-72 hours; we then switch to maintenance doses. 
Consideration may be given to administering $200 \mathrm{mg}$ immediately at zero hour (without a time interval) for even faster saturation, then every 6 hours as above.

Maintenance Treatment: Maintenance dose treatment should last for quite a long period of time. There is no golden mean, and the dose may need to be changed on a case-tocase basis. The maintenance treatment usually takes from 7 to 8 days, but sometimes even from 10 to 14 days, depending on how long the disease has been lasting and on when amantadine was incorporated as part of the treatment.

\section{In case of no clear health improvement}

If the patient exhibits no spectacular health improvement after 48 hours, and there is yet no stabilization, it can be suspected that the patient is already suffering from some complications, such as pneumonia, for example. Such a patient must be examined and absolutely auscultated. Lesions detectable through auscultation require antibiotic therapy, best through the administration of two antibiotics together, be it intramuscularly or intravenously. Steroidal drugs are not to be administered if not needed, unless they must be used in critical cases. Any interstitial lesions visible thanks to sectional roentgenography scanning should regress after treatment involving the use of amantadine.

\section{Results}

\section{Demographic and clinical characteristics}

A total of 100 patients with clinical symptoms compatible with SARS-Cov-2 were included in this study. Table 1 shows the characteristics of the patients and the symptomatology they presented. 100 middle-aged patients were studied. Most of them presented a state of weakness, loss of appetite, body pain, fever and cough.

The treatment with amantadine was started from the first day of the examination. Figure 1 shows the number of patients

Table 1: Characteristics of the patients and Symptoms.

\begin{tabular}{|l|l|}
\hline Variable & Patients $n=100$ \\
\hline Gender, $\mathrm{n}(\%)$ & Male 60, Female 40 \\
\hline Age (years), mean \pm SD & $\begin{array}{l}10-90, \mathrm{~F}(56.11 \pm 14.50), \\
\mathrm{M}(56.09 \pm 14.26)\end{array}$ \\
\hline Weight (kg) & $44-120$ \\
\hline Height (cm) & $143-188$ \\
\hline BMI & $17.99-39.78$ \\
\hline Symptoms (\%) & 79 \\
\hline Fever & 77 \\
\hline Cough & 38 \\
\hline Dyspnea & 42 \\
\hline Chest Pain & 53 \\
\hline Back Pain & 78 \\
\hline Body Pain & 38 \\
\hline Diarrhea & 94 \\
\hline Weakness & 80 \\
\hline Loss of appetite & 67 \\
\hline Loss of smell and taste & 43 \\
\hline Pneumonia & 55 \\
\hline RT-PCR+SARS-Cov-2 & \\
\hline
\end{tabular}

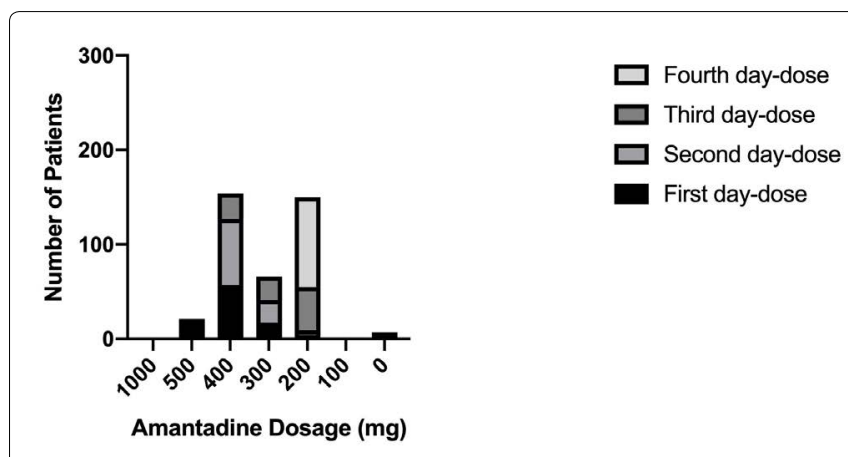

Figure 1: Dosage of amantadine used on patients during the first 4 days.

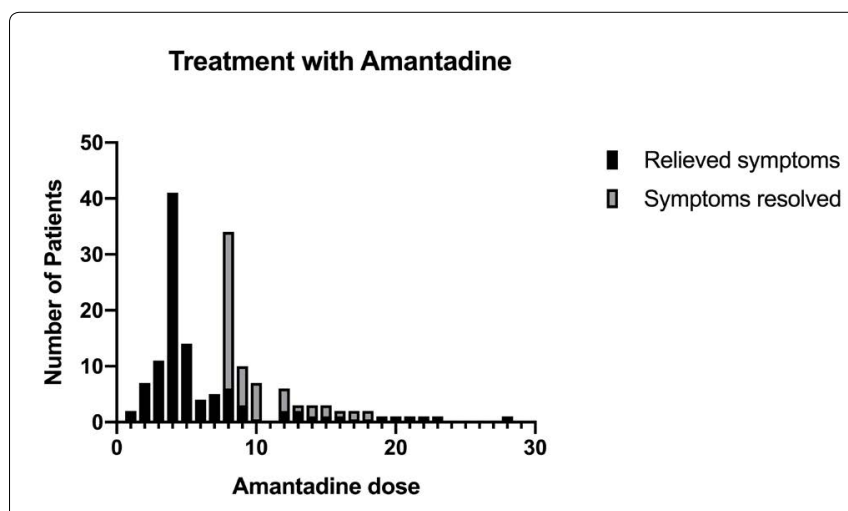

Figure 2: Amantadine dose until symptoms are relieved and resolved.

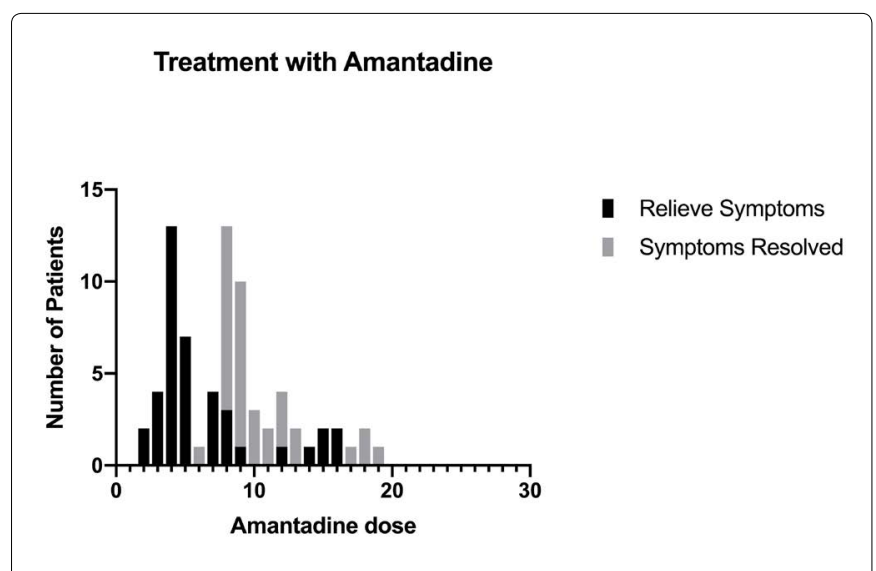

Figure 3: Amantadine dose in patients with pneumonia.

who were treated with different initial doses of amantadine. The first dose consisted de $500 \mathrm{mg}$ of amantadine, depending on the patient's symptoms at that moment, with the aim of stabilizing the patient as soon as possible. The second and third doses were between 200 and $400 \mathrm{mg}$ of amantadine and the fourth and subsequent doses were $200 \mathrm{mg}$. Figure 2 shows the doses in which the main symptoms of SARSCov-2 disease were alleviated, most patients responded satisfactorily to the fourth dose of treatment, with this we can determine that clinically the virus stops generating symptoms. From the eighth doses most patients did not present any symptoms, indicating that treatment with amantadine is satisfactory to decrease the effects that SARS-Cov-2 causes in infected persons. Figure 3 shows the 
patients who became complicated with pneumonia but were treated with amantadine and the antibiotic azithromycin. Azithromycin was used as protection in most cases. In the pneumonia the combination of gentamicin and cefuroxime was used depending on the severity, most of these patients began to lose symptoms on the seventh day of treatment.

\section{Discussion}

Early treatment is recommended because it reduces the time of the negative impact of the virus on the body and alleviates later complications. If the patient has the specific symptoms of the disease, we can suspect a COVID-19 infection with a high probability rate. The opinion is that treatment involving the use of amantadine hydrochloride should be implemented without waiting for the test results. The test should be taken independently of the treatment. If the test result is positive and the patient suffers from the symptoms, the treatment should be applied immediately. Amantadine is safe to use, however some side effects have occurred in some persons.

In the majority of cases, patients feel dryness in the oral cavity, which in no way constitutes a contraindication to the treatment. When administering amantadine hydrochloride, we recommend giving large quantities of liquids to drink, from 3 to 4 litres, at least at the first stage of intense treatment.

Approximately $10 \%$ of patients may experience mild anxiety, periodical agitation or a sleep disorder in 3-5 days from implementation of the treatment.

In case of a sleep disorder, it is recommended to administer a sleeping drug - which should be perfectly sufficient. The patient's body rests in sleep, fully recovering at this time. The symptoms subside, or they at least do not get more intense.

It is important to mention that the use of amantadine has been used to treat COVID-19 $[5,7]$, it has also been observed that patients with Parkinson's disease [8] and multiple sclerosis [9] who take amantadine and for some reason became infected by coronavirus, do not develop the symptomatology. Some patients were treated with amantadine and azithromycin, this antibiotic is able to reduce the exaggerated production of macrophages and cytokines [10] pro-inflammatory, as well as the prevention of bacterial aggregation generated in the respiratory processes [11].

\section{Conclusion}

Amantadine, the main drug (active substance) we are considering, is not included in COVID-19 treatment regimens for now, although it is a drug known about for 30-40 years and is used to treat other diseases. It is well-tested and known worldwide. With the use of it, we are already talking about causal treatment - that is, complete deactivation of the virus. It no longer works on the body, which is why the therapeutic effect is so much improved.

Once a virus has been deactivated, it will never attempt to replicate again. It takes at least 24 hours for the drug to enter the body in sufficient quantities and to change the environment for the virus.

Based on my experiences, I expect that this drug will be immediately implemented in COVID-19 treatment scheme and may revolutionize the fight against the pandemic worldwide, saving the health and life of millions.

No conflict of interest.

\section{References}

1. Abreu GEA, Aguilar MEH, Covarrubias DH, et al. (2020) Amantadine as a drug to mitigate the effects of COVID-19. Med Hypotheses 140: 109755.

2. Araújo R, Aranda-Martínez JD, Aranda-Abreu GE (2020) Amantadine Treatment for People with COVID-19. Arch Med Res 51: 739-740.

3. Mandala VS, McKay MJ, Shcherbakov AA, et al. (2020) Structure and drug binding of the SARS-CoV-2 envelope protein transmembrane domain in lipid bilayers. Nat Struct Mol Biol 27: 1202-1208.

4. Sun P, Lu X, Xu C, et al. (2020) Understanding of COVID-19 based on current evidence. J Med Virol 92: 548-551.

5. Aranda-Abreu GE, Aranda-Martínez JD, Araújo R, et al. (2020) Observational study of people infected with SARS-Cov-2, treated with amantadine. Pharmacol Rep 10: 1-4.

6. von Elm E, Altman DG, Egger M, et al. (2008) The Strengthening the Reporting of Observational Studies in Epidemiology (STROBE) statement: Guidelines for reporting observational studies. J Clin Epidemiol 61: 344-349.

7. Aranda-Abreu GE, Aranda-Martínez JD, Araújo R (2020) Use of amantadine in a patient with SARS-CoV-2. J Med Virol 93: 110111.

8. Cortés Borra A (2020) Does amantadine have a protective effect against COVID-19. Neurol Neurochir Pol 54: 284-285.

9. Rejdak K, Grieb P (2020) Adamantanes might be protective from COVID-19 in patients with neurological diseases: Multiple sclerosis, parkinsonism and cognitive impairment. Mult Scler Relat Disord 42: 102163.

10. Meyer M, HuauxF, Gavilanes X, et al. (2009) Azithromycin reduces exaggerated cytokine production by $\mathrm{M} 1$ alveolar macrophages in cystic fibrosis. Am J Respir Cell Mol Biol 41: 590-602.

11. Welte T (2019) Azithromycin: The holy grail to prevent exacerbations in chronic respiratory disease. Am J Respir Crit Care Med 200: 269-270.

DOI: $10.36959 / 719 / 584$

Copyright: (C) 2021 Bodnar W. This is an open-access article distributed under the terms of the Creative Commons Attribution License, which permits unrestricted use, distribution, and reproduction in any medium, provided the original author and source are credited. 